\title{
Assessment of Low-Level Laser Therapy Effects After Extraction of Impacted Lower Third Molar Surgery
}

\author{
Shahrokh Raiesian', Mehdi Khani² ${ }^{*}$, Kazem Khiabani' ${ }^{1}$ Ershad Hemmati², Mohammad Pouretezad ${ }^{3}$ \\ ${ }^{1}$ Department of Oral and Maxillofacial Surgery, Faculty of Dentistry, Ahvaz Jundishapur University of Medical Sciences, \\ Ahvaz, Iran \\ ${ }^{2}$ Department of Oral and Maxillofacial Surgery, Faculty of Dentistry, Jundishapur University of Medical Sciences, Ahvaz, Iran \\ ${ }^{3}$ Department of Physiotherapy, Faculty of Rehabilitation, Jundishapur University of Medical Sciences, Ahvaz, Iran
}

*Correspondence to Mehdi Khani, MD; Department of Oral and Maxillofacial Surgery, Faculty of Dentistry, Ahvaz JundishapurUniversity of Medical Sciences, Ahvaz, Iran.

Tel: +98-6133205168; Fax: +98-2122749221; Email:

dr.khani6182@gmail.com

Published online 19 December 2016

Introduction

Third molar surgery is almost one of the most frequent procedures performed by maxillofacial surgeons. The surgical trauma leads to post-operation complications such as pain, inflammation and trismus. ${ }^{1}$ About 3-5 hours following surgery, the pain reaches its maximum intensity and may last 2-3 days; and then diminishes within 7 days after surgery. ${ }^{2,3}$ Moreover, post-operative inflammation disappears 5-7 days after surgery. ${ }^{4}$ It has been recommended to use local or systemic steroid and non-steroid anti-inflammatory therapy to reduce inflammation and relieve pain after molar surgery, but these drugs present some side effects, including gastrointestinal issues, systemic bleeding and allergic reactions. ${ }^{5}$ Several studies have demonstrated that laser therapy can accelerate cell and tissue reconstruction as well as relieve post-operative pain. ${ }^{5,6}$ Laser therapy is an open research field. However, some studies have shown useful findings in treatment of dentin hypersensitivity, temporomandibular joint disorders, inferior alveolar nerve paraesthesia resulting from third molar surgery, sagittal osteotomy, trigeminal neuralgia, labial herpes, aphthous ulcers and post-chemotherapy and ray inflammation. ${ }^{7-12}$ As there are conflicting findings regarding the effectiveness of low-level laser therapy (LLLT) and the importance of controlling third molar surgery's complications, this study aimed to assess the effect of LLLT on pain, swelling and maximum mouth opening in patients undergoing third molar surgery. In this study, we increased the number and variety of studied groups in order to reduce the error rate, in comparison to former researches. To eliminate the possible differences between several people in relevance to pain amount and also their reports on it, a bilateral surgery group was used to study the effects of LLLT on the post-operation pain and swelling. On the other hand, as maximum mouth

Please cite this article as follows: Raiesian S, Khani M, Khiabani K, Hemmati E, Pouretezad M. Assessment of low-level laser therapy effects after extraction of impacted lower third molar surgery. J Lasers Med Sci. 2017;8(1):42-45. doi:10.15171/jlms.2016.08. 
opening can be limited by unilateral pain, two distinct groups were considered for trismus.

\section{Methods}

This study is a clinical trial that was conducted on 44 patients at the Dental School at Ahvaz Jundishapur University of Medical Sciences, in 2015. The inclusion criteria included a range of age from 18 to 30 years old, absence of systemic diseases and impacted third molar with III B difficulty degree (according to Winter's and Gregory's classifications). ${ }^{13}$ Exclusion criteria consisted of contraindications for laser therapy, teeth with pulp involvement, smoking, oral contraception, pregnancy and breast feeding.

All patients underwent a standard surgery procedure. The operator who performed the LLLT in all patients of the experimental group, was different from the surgeon. Another operator carried out blind measurements in both control and experimental groups. Bilateral surgery was performed on 15 patients, for whom one operated side received LLLT, and the other side a hand piece without laser was placed over surgical site. In addition, 13 patients underwent unilateral laser therapy and 16 patients did not receive laser therapy at all, for trismus evaluation. Patients were randomly divided into two groups of intervention (28 cases) and control (31 cases). The patients were requested to not use any analgesic drugs at least 12 hours before surgery. The patients in the intervention group received laser therapy, while patients in the control group received routine treatment. The laser was administrated intra-orally on 2 points of vestibular and lingual sites at 1 $\mathrm{cm}$ from surgery site, and extra-orally at the emergence of the masseter muscle immediately after surgery, as well as 24 hours after surgery. After surgery, all patients received 1 gr amoxicillin orally per 12 hours up to 5 days and 1600 mg ibuprofen up to 2 days. In this study, we utilized diode, fixed and contact mode laser (G-laser 25, Galbiati, Italy) with a continuous wavelength of $980 \mathrm{~nm}$, with a hand piece of $600 \mu \mathrm{m}$. The laser energy was justified on 300 $\mathrm{mW}$ and emitted for 180 seconds (60 seconds for each site) $(0.3 \mathrm{~W} \times 60 \mathrm{~s}=18 \mathrm{~J}) .{ }^{13}$ The amount of mouth opening between lower and upper right central incisors before surgery (baseline) and after surgery was measured using Collis. Moreover, the amount of swelling was obtained by measuring to the distance between the mandibular angle and pogonion, the mandibular angle and the ipsilateral ala, the mandibular angle and the lateral canthus of the ipsilateral eye and also the distance between mandibular angle and tragus, before surgery, 24 hours and a week after surgery. The patients were recommended to complete a questionnaire with visual analogue scale (VAS) to measure pain intensity and its duration.

\section{Results}

Totally, 28 patients in the laser therapy group were analyzed. They were in the age range of 18-44 years and the mean age was $27.4 \pm 5.5$ years. Of those, 14 subjects $(50 \%)$ were males. The mean age in the 2 groups is shown in Table 1. 16patients were in the routine therapy group with an age range of 21-36 years and mean age 27.4 5.5 years. The mean age between laser therapy and drug groups did not significantly differ $(P<0.0001)$. Moreover, of 16 cases in the drug group, 8 (50\%) were females.

The pain score in the 2 groups was measured twice: 24 hours and 1 week after surgery. The mean score of pain at 24 hours after surgery in the laser therapy group $(2.3 \pm 3.5)$ was significantly lower than in the drug therapy group $(4.19 \pm 3.09)(P=0.036)$. Moreover, the mean score of pain at one week after surgery in laser therapy group $(0.13 \pm 2.33)$ was significantly lower than drug therapy group $(1.43 \pm 2.45)(P=0.046$; Table 2$)$.

The amount of swelling was obtained by measuring the distance between the mandibular angle and lateral canthus of eye, 24 hours and one week after surgery. There was no significant difference for mean swelling between 24 hours and 1 week after surgery. These findings are presented in Table 3.

The amount of swelling measured between mandibular angle and ala at 24 hours after surgery was similar between laser and drug therapy groups $(5.64 \pm 3.9$ and $5 \pm 4.38$, respectively, $P=0.5)$. Similarly, the results were the same at 1 week after surgery $(P=0.12$; Table 4$)$.

The mean difference score of swelling, measured from the angle to lip commissure is presented in Table 5. This finding did not show statistically significant difference between the 2 groups neither at 24 hours nor at 1 week after surgery $(P=0.356$ and $P=0.795$, respectively).

There was no significant difference of swelling between the angle and pogonion for the laser therapy $(4.43 \pm 5.39)$ and drug therapy groups $(4.88 \pm 4.5)$ at 24 hours after surgery $(P=0.504)$. Moreover, this value remained similar between the 2 groups at 1 week after surgery $(P=0.168$; Table 6).

The mean difference score of swelling from the angle to

Table 1. Mean Age Score in 2 Groups

\begin{tabular}{llllll}
\hline & Group & Number & Mean & SD & P Value \\
\hline \multirow{2}{*}{ Age } & Laser & 28 & 27.46 & 5.588 & \multirow{2}{*}{0.619} \\
& Drug & 16 & 28.44 & 7.183 & \\
\hline
\end{tabular}

Table 2. The Comparison Between Mean Score of Pain Between 2 Groups

\begin{tabular}{llllll}
\hline & Group & Count & Mean & SD & P Value \\
\hline Pain at 24 h after & Laser & 16 & 2.31 & 3.55 & \multirow{2}{*}{0.036} \\
surgery & Drug & 28 & 4.19 & 3.09 & \\
Pain at 1 wk after & Laser & 16 & 0.13 & 2.33 & \multirow{2}{*}{ surgery } \\
\cline { 1 - 1 }
\end{tabular}

Table 3. The Mean Difference Score of Swelling From the Angle to Lateral Canthus of Eye at 24 Hours and 1 Week After Surgery

\begin{tabular}{llllll}
\hline & Group & Count & Mean & SD & P Value \\
\hline Swelling at 24 h & Laser & 28 & 2.43 & 2.78 & \multirow{2}{*}{0.309} \\
after surgery & Drug & 16 & 3.44 & 3.97 & \\
Swelling at 1 wk & Laser & 28 & 0.43 & 2.60 & \multirow{2}{*}{0.13} \\
after surgery & Drug & 16 & 0.31 & 2.21 & \\
\hline
\end{tabular}


tragus is demonstrated in Table 7. The mean difference score of swelling did not significantly differ either at 24 hours or at 1 week after surgery between the 2 groups $(P=0.305$ and 0.388 , respectively).

The mean difference score of MMO in the laser therapy group (-13.6) did not significantly differ with the drug therapy group $(-13.94)$ at 24 hours after surgery $(P=0.7)$. In addition, this value did not significantly differ between the 2 groups $(P=0.75$; Table 8$)$.

\section{Discussion}

Considering the inconsistent findings regarding the effectiveness of LLLT and the importance of complication management after third molar surgery, the aim of this

Table 4. The Mean Difference Score of Swelling From the Angle to Ala Measurement at 24 Hours and 1 Week After Surgery

\begin{tabular}{llllll}
\hline & Group & Count & Mean & SD & P Value \\
\hline Swelling at 24 h & Laser & 28 & 5.64 & 3.90 & \multirow{2}{*}{0.526} \\
after surgery & Drug & 16 & 5.00 & 4.38 & \\
Swelling at 1 wk & Laser & 28 & 1.21 & 2.11 & \multirow{2}{*}{0.12} \\
after surgery & Drug & 16 & 0.00 & 5.16 & \\
\hline
\end{tabular}

Table 5. The Mean Difference Score of Swelling Between the Angle and Lip Commissure at 24 Hours and 1 Week After Surgery

\begin{tabular}{llllll}
\hline & Group & Count & Mean & SD & P Value \\
\hline Swelling at 24 h & Laser & 28 & 7.68 & 7.33 & \multirow{2}{*}{0.356} \\
after surgery & Drug & 16 & 9.13 & 6.48 & \\
Swelling at 1 wk & Laser & 28 & 2.89 & 5.90 & \multirow{2}{*}{0.795} \\
after surgery & Drug & 16 & 1.25 & 6.71 & \\
\hline
\end{tabular}

Table 6. The Mean Difference Score of Swelling the Angle to Pogonion at 24 Hours and 1 Week After Surgery

\begin{tabular}{llllll}
\hline & Group & Count & Mean & SD & P Value \\
\hline Swelling at 24 h & Laser & 28 & 4.43 & 5.39 & \multirow{2}{*}{0.504} \\
after surgery & Drug & 16 & 4.88 & 4.50 & \\
Swelling at 1 wk & Laser & 28 & 1.46 & 3.14 & \multirow{2}{*}{0.168} \\
after surgery & Drug & 16 & 1.25 & 5.92 & \\
\hline
\end{tabular}

Table 7. The Mean Score of Swelling From the Angle to Tragus at 24 Hours and 1 Week After Surgery

\begin{tabular}{llllll}
\hline & Group & Count & Mean & SD & P Value \\
\hline Swelling at 24 h & Laser & 28 & 2.79 & 3.67 & \multirow{2}{*}{0.305} \\
after surgery & Drug & 16 & 1.25 & 2.70 & \\
Swelling at 1 wk & Laser & 28 & 0.79 & 2.54 & \multirow{2}{*}{0.388} \\
after surgery & Drug & 16 & 0.00 & 4.47 & \\
\hline
\end{tabular}

Table 8. The Mean Difference Score of MMO at 24 Hours and 1 Week After Surgery

\begin{tabular}{llllll}
\hline & Group & Count & Mean & SD & P Value \\
\hline MMO changes & Laser & 28 & -13.64 & 9.29 & \multirow{2}{*}{0.759} \\
after 24 h & Drug & 16 & -13.94 & 12.91 & \\
MMO changes & Laser & 28 & -7.75 & 9.03 & \multirow{2}{*}{0.758} \\
after 1 wk & Drug & 16 & -8.44 & 11.20 & \\
\hline
\end{tabular}

Abbreviation: MMO, Maximum Mouth Opening study was to assess the effect of LLLT on reducing pain and the maximum mouth opening after third molar surgery.

The current study showed that the amount of swelling according to different measurements did not significantly differ between the two groups, neither 24 hours nor 1 week after surgery. However, the mean score of pain in the laser therapy group was significantly lower than in the drug therapy group at 24 hours and also one week after surgery. These findings are consistent with the study carried out by Brignardello-Petersen et al, which have evaluated the effectiveness and safety of low-level laser diode with a wavelength range of 610-810 $\mathrm{nm}$ and a laser energy range of 10-500 $\mathrm{mW}$, for pain and swelling reduction and MMO increase after impacted third molar surgery. They have shown that laser therapy does not affect pain and swelling, but slightly improved MMO factor. ${ }^{14}$ In a clinical trial, the assessment of analgesia and anti-inflammatory effects of low-level laser has revealed that it has no effect on swelling and pain reduction and improvement of trismus after impacted third molar surgery. ${ }^{15}$ In another study, laser therapy could slightly, but not significantly, reduce pain, swelling and trismus. ${ }^{16}$

Some studies have shown that LLLT has useful impact on the management of pain and swelling. ${ }^{14,15,17}$ In a study done by Ferrante et al, it has been reported that laser therapy is useful for pain reduction in impacted third molar surgery. ${ }^{13}$ In an animal study, it has been shown that LLLT could improve pain intensity, inflammation and immunologic reactions; while in human studies some factors including the type of disease, surgery and laser related variables impact the outcome of laser therapy. ${ }^{17}$ In a study carried out by Sanz-Moliner et al, it has been demonstrated that laser therapy can diminish pain and reduce swelling compared to control group in patients with severe chronic periodontitis who underwent flap surgery. ${ }^{18}$ Aras and Güngörmüş have compared the effectiveness of intraoral and extraoral laser administration. They reported that extraoral laser therapy has better effects on improving pain intensity and trsimus after impacted third molar surgery. ${ }^{19}$ Modulation of inflammatory process and relieve of acute pain in the short period by LLLT is well-demonstrated. LLLT energy absorption by tissues and interaction of its photons with cellular structures cause partial production of LLLT biological effects. Expected therapeutic effects arise from this interaction. Pain relief, wound healing and muscle relaxation are results of increased cellular energy and changes in cell membrane permeability. LLLT favors the hyperpolarized state, directly over primary nerve endings, that inhibits transmission of painful stimuli to central nervous system. ${ }^{20}$ LLLT has significant and rapid effects in reducing the level of pain and inflammation mediators such as prostaglandin E2 (PGE2), interleukin 1 (IL1), tumor necrosis factor (TNF) and cyclooxygenase 2 (COX 2$){ }^{21}$

Finally, according to our findings and previous studies, it seems that other effective factors, complications, type and method of laser therapy need to be evaluated in 
future studies. In addition, a large sample size with longer follow-up period is recommended.

\section{Conclusion}

Our findings showed that LLLT was useful in diminishing pain and could slightly reduce the swelling in comparison with drug therapy, in impacted third molar surgery.

\section{Ethical Considerations}

The study was approved by the ethical committee of Ahvaz Jundishapur University of Medical Sciences (IRCT2016061228402N1). All participants signed an informed consent form.

\section{Conflict of Interests}

Authors declare that they have no conflict of interests.

\section{References}

1. Grossi GB, Maiorana C, Garramone RA, Borgonovo A, Creminelli L, Santoro F. Assessing postoperative discomfort after third molar surgery: a prospective study. $J$ Oral Maxillofac Surg. 2007;65(5):901-17.

2. Lago-Méndez L, Diniz-Freitas M, Senra-Rivera C, GudeSampedro F, Rey JMG, García-García A. Relationships between surgical difficulty and postoperative pain in lower third molar extractions. J Oral Maxillofac Surg. 2007;65(5):979-83.

3. Marković AB, Todorović L. Postoperative analgesia after lower third molar surgery: contribution of the use of longacting local anesthetics, low-power laser, and diclofenac. Oral Surg Oral Med Oral Pathol Oral Radiol Endodontol. 2006;102(5):e4-e8.

4. Markovic A, Todorovic L. Effectiveness of dexamethasone and low-power laser in minimizing oedema after third molar surgery: a clinical trial. J Oral Maxillofac Surg. 2007;36(3):226-229.

5. Kreisler M, Al Haj H, Noroozi N, Willershausen B, d'Hoedt B. Efficacy of low level laser therapy in reducing postoperative pain after endodontic surgery-a randomized double blind clinical study. J Oral Maxillofac Surg. 2004;33(1):38-41.

6. Coluzzi DJ, Convissar RA. Lasers in clinical dentistry. Int $J$ Dent Clin. 2004;48(4):11-2.

7. Arora H, Pai KM, Maiya A, Vidyasagar M, Rajeev A. Efficacy of He-Ne Laser in the prevention and treatment of radiotherapy-induced oral mucositis in oral cancer patients. Oral Surg Oral Med Oral Pathol Oral Radiol Endod. 2008;105(2):180-6. e181. doi:10.1016/j.tripleo.2007.07.043.

8. Fikácková $H$, Dostálová $T$, Navrátil L, Klaschka J. Effectiveness of low-level laser therapy in temporomandibular joint disorders: a placebo-controlled study. Photomed Laser Surg. 2007;25(4):297-303.

9. Marsilio AL, Rodrigues JR, Borges AB. Effect of the clinical application of the GaAlAs laser in the treatment of dentine hypersensitivity. J Clin Laser Med Surg. 2003;21(5):291-296.

10. Miloro M, Repaskyb M. Low-level laser effect on neurosensory recovery after sagittal ramus osteotomy. Oral Surg Oral Med Oral Pathol Oral Radiol Endod. 2000;89(1): 12-18.

11. Ozen T, Orhan K, Gorur I, Ozturk A. Efficacy of low level laser therapy on neurosensory recovery after injury to the inferior alveolar nerve. Head Face Med. 2006;2(1):1.

12. Parker S. Verifiable CPD paper: Low-level laser use in dentistry. Br Dent J. 2007;202(3):131-138.

13. Ferrante M, Petrini M, Trentini P, Perfetti G, Spoto G. Effect of low-level laser therapy after extraction of impacted lower third molars. Lasers Med Sci. 2013;28(3):845-849. doi: 10.1007/s10103-012-1174-4.

14. Brignardello-Petersen R, Carrasco-Labra A, Araya I, Yanine N, Beyene J, Shah PS. Is adjuvant laser therapy effective for preventing pain, swelling, and trismus after surgical removal of impacted mandibular third molars? A systematic review and meta-analysis. J Oral Maxillofac Surg. 2012;70(8):1789-1801. doi: 10.1016/j.joms.2012.01.008.

15. López-Ramírez M, Vílchez-Pérez MÁ, Gargallo-Albiol J, Arnabat-Domínguez J, Gay-Escoda C. Efficacy of low-level laser therapy in the management of pain, facial swelling, and postoperative trismus after a lower third molar extraction. A preliminary study. Lasers Med Sci. 2012;27(3):559-566. doi: 10.1007/s10103-011-0936-8.

16. Amarillas-Escobar ED, Toranzo-Fernández JM, MartínezRider R, Noyola-Frías MA, Hidalgo-Hurtado JA, Serna VMF, et al. Use of therapeutic laser after surgical removal of impacted lower third molars. J Oral Maxillofac Surg. 2010; 68(2):319-324. doi: 10.1016/j.joms.2009.07.037.

17. Fekrazad R, Chiniforush N, Bouraima SA, Valipour M, Aslani M, Zare M, Safari OA. Low level laser therapy in management of complications after intra oral surgeries. $J$ Lasers Med Sci. 2012;3(4):135.

18. Sanz-Moliner JD, Nart J, Cohen RE, Ciancio SG. The effect of an 810-nm diode laser on postoperative pain and tissue response after modified Widman flap surgery: a pilot study in humans. J Periodontol. 2013;84(2):152-158. doi: 10.1902/ jop.2012.110660.

19. Aras MH, Güngörmüş M. Placebo-controlled randomized clinical trial of the effect two different low-level laser therapies (LLLT)--intraoral and extraoral--on trismus and facial swelling following surgical extraction of the lower third molar. Lasers Med Sci. 2010;25(5):641-645. doi: 10.1007/s10103-009-0684-1.

20. Carroll JD, Milward MR, Cooper PR, Hadis M, Palin WM. Developments in low level light therapy (LLLT) for dentistry. Dent Mater. 2014;30(5):465-475.

21. Bjordal JM, Johnson MI, Iversen V, Aimbire F, LopesMartins RAB. Low-level laser therapy in acute pain: a systemic review of possible mechanisms of action and clinical effects in randomized placebo-controlled trials. Photochem Laser Surg. 2006;24(2):158-168. 